Manuscript ID: 20-255-1-ED

"Development of a Smoke-free Homes Intervention for Parents: An Intervention Mapping Approach"

\title{
Response to reviewers' comments
}

We are extremely grateful to the reviewers for their time and effort in reviewing this manuscript and for recognising the importance of this work. The comments have been most helpful in our revision of this work. We provide detailed responses to each point in the document below and have used tracked changes to the initial manuscript to assist with the re-review.

\section{Editorial revision requests:}

Major revisions:

Reviewer A suggests that you could expand your review on second-hand smoke in homes, and clarify the fit with existing projects.

Response:

We have expanded our review of literature regarding interventions designed to reduce secondhand smoke exposure in the home to assist the reader gain a sense of how the current project fits with previous research. In addition, we have added clarity on the fit of the current project with existing research in this area, and how it builds on previous research utilising personalised air quality feedback, being designed to be used with parents who wish to move towards having a smoke-free home AND other household members.

Both reviewers thought more information on the focus group process would benefit the paper, and there were also some suggestions around the relationship between the paper and the supplementary materials.

Response

We have provided additional information on the focus group process as requested, including an outline of the design and procedures for recruitment, data collection and analysis. We have also clarified throughout the paper where decisions/choices have been made based on the literature review work outlined in supplementary materials.

Reviewer $B$ also has a number of suggestions and questions in relation to IM.

We have provided detailed descriptions of the ways in which we have addressed Reviewer B's suggestions/questions related to IM from page 5 onwards.

\section{Reviewer A:}

Section 1a, Full Disclosure: Replication Package: I could not see the above that have not been ticked. Analysis plan is not required and inclusion of the ethical approval proposal for the qualitative work seems unnecessary. 
The documents within the replication package were uploaded on the basis of the submission guidance on the Health Psychology Bulletin website, which states that the replication package should contain enough information to enable a third party to replicate the study. The guidance includes uploading the ethics application and related documents.

Section 1b, Mandatory pre-registration components: Most of these are not relevant. I didn't see a disclosure of interest

Thank you for pointing this out. We have included a disclosure of interest statement on the front page of the resubmitted article.

Section 2a: A large amount of work has clearly gone into this project and it looks very systematic and of high quality. I do not question the quality of the intervention development work. However, the manuscript doesn't read like a journal article. It reads like a thesis chapter. Also, if the formative work represents 'research', then much more detail about these are required. For example, the qualitative work in step 4 has a few lines describing the methods. If the editors agree (I am not fully sure of the format for HPB articles) then I suggest the authors revise the manuscript so it is more like a journal article. I have made comments below.

Response:

We thank the reviewer for their positive feedback on the quality of the intervention development work. We acknowledge that the manuscript doesn't read like a traditional journal article. It describes in detail how we used the Intervention Mapping methodology to develop a theory-and evidence-based smoke-free homes intervention programme. The format of this article is based on existing published papers outlining the development of behaviour change interventions in other areas of health promotion using intervention mapping. The intervention mapping papers we have read do present less detail than a traditional journal article would on the research elements of the development process, to enable a full description of the intervention development process as a whole within or near to given word counts. In addition, they are formatted differently to traditional journal articles, given their focus on describing in detail the step-by-step development of theory-and evidence-based intervention programmes. We have provided additional detail on the qualitative work undertaken as part of Step 4, including an outline of the design and procedures for recruitment, data collection and analysis. However, given the nature of this project, and already published articles taking the same approach to intervention development, we feel it would not be appropriate to restructure the manuscript so that it is more like a journal article.

Abstract - I felt the abstract could be improved if some sense of how the systematic development/formative studies helped shape the intervention.

Response:

Thank you for your suggestion. We have revised the abstract to provide examples of how the literature review, focus group/interview findings and panel group discussions informed the development of the intervention.

Introduction - the existing literature on reducing SHS in homes should be summarised to help the reader get a sense of where this project fits in. The rationale for the current study was also not clear 
- the authors report that the REFRESH intervention (or one very similar to it) had been piloted and tested in an RCT, but no sense of whether it had a positive or negative impact. If a positive or likely positive impact, then a clear rationale for why the intervention is being revised should be provided. Simply to inject more theory into it doesn't seem like a very strong rationale currently.

Response:

We have expanded our review of literature regarding interventions designed to reduce secondhand smoke exposure in the home to assist the reader gain a sense of how the current project fits with previous research. In addition, we have added clarity on the fit of the current project with existing research in this area, and how it builds on previous research utilising personalised air quality feedback, being designed to be used with parents who wish to move towards having a smoke-free home AND other household members.

Rapid reviews - it is difficult to assess how these were undertaken from the manuscript. I find it unusual to have a supplementary document which is like a separate paper reporting on the process and findings. The manuscript would ideally be a complete work, with supplementary documents providing additional rather than required information.

Response:

We acknowledge that the manuscript would ideally be one complete work, however given the quantity of work undertaken during the intervention mapping process to develop a theory and evidence-based intervention, and given the existing word-count, providing a full account of the different processes undertaken for the three reviews within the manuscript is indeed a challenge. It is not unusual for intervention mapping papers to provide the detail of literature reviews undertaken in supplementary documents, even when only one review is conducted, as this then enables the detail of subsequent intervention mapping steps, and the presentation of the intervention itself, to be incorporated into the manuscript.

IM step 3 - the authors refer to 'behaviour change intervention methods' but it is unclear what this refers to. It seems to include methods of delivery e.g. discussion, and techniques e.g. goal setting. I suggest they align with the behaviour change technique taxonomy v1 (Michie et al, 2013) where possible. If intervention methods are part of IM (which I am not very familiar with) and these are described elsewhere then perhaps the authors could reference this to help readers like myself?

Response:

Stage 3 of the intervention mapping process involves selecting appropriate theoretical methods to change behaviour and translating these into practical strategies. For each determinant (i.e. knowledge), appropriate theoretical methods are identified using a taxonomy, combined with existing literature. Michie et al's behaviour change technique taxonomy has been suggested to be better suited to intervention coding than intervention development (see Kok et al 2016), and on this basis we used Bartholomew's taxonomy for intervention development, which was published by Kok et al (2016) and extracted from the Intervention Mapping protocol (Bartholomew et al 2011). Our choice of taxonomy is stated in paragraph 1 , under the 'Methods' section of Step 3.

IM step 4-details of the methods of the interviews and focus groups is required, unless this is published elsewhere and then a link to that publication is needed. Otherwise this will require a great deal more information about the design, recruitment, data collection, analysis etc. 


\section{Response:}

We have provided additional detail on the nature of the discussions that took place at the focus groups and during the interviews. We have also added text to clarify the procedures for the focus groups and interviews, and who was present. The topic guide/interview schedule are also now included as supplementary materials. Providing a great deal more information within the main body of the article is a challenge given these were just two components of the overall development process, and we are following a format that is more usual for intervention mapping papers - providing an outline of the interviews and focus groups element of the work, whilst enabling the detail of other IM steps and the presentation of the intervention itself to be incorporated into the manuscript.

There are a lot of tables and figures to do with the intervention components and development process, but none on the actual data that informed the intervention as far as I can see (other than the supplementary documents, though presumably these won't be included in the main manuscript). Suggest the authors consider what the data is that they are using and include a summary of this in the paper.

The introduction and discussion are very short. Again, not written like a typical journal article.

Thank you for your suggestions. We acknowledge that the manuscript doesn't read like a typical journal article. As outlined on page 2, the format of this article is based on existing published papers outlining the development of behaviour change interventions in other areas of health promotion using intervention mapping. The intervention mapping papers we have read do present less detail than a traditional journal article would on the research elements of the development process, and use figures and tables to describe the intervention development process using the same approach that we have. Intervention mapping journal article are formatted differently to traditional journal articles, given their focus on describing in detail the step-by-step development of theory-and evidence-based intervention programmes. We have provided additional detail in the introduction section in relation to your earlier comment (see page 1). We have also added additional information on the interviews/focus groups that contributed to informing the development process, and have included additional supplementary materials related to the rapid reviews undertaken. We have also clarified throughout the text where our review work and/or interviews/focus group findings contributed to our choices made during the development process.

\section{Reviewer B}

Section 1a, Full Disclosure: Replication Package: The current paper addresses the planned and systematic development of an intervention to reduce second hand-smoking. The elements described above are not all relevant. However, in order to guide the development process, the authors have engaged in small-scaled studies, like focus groups. It might be good to add the materials used for these studies as additional files (e.g. interview guides, result summary).

Thank you for your suggestion. The focus group topic guide and interview schedule were already included in our replication package. We have now also included a summary of findings from the focus groups and interviews.

Section 2a: This paper addresses a very important topic, namely exposure to second-hand smoke, which is associated with various adverse outcomes. In order to deal with this issue, it is important to develop interventions that are focused on creating a smoke-free homes. The current paper gives clear insights into the process of developing such an intervention using the Intervention Mapping 
approach. Clearly describing this process, increases transparency and gives insights into the choices that were made and the evidence used to support these choices; something which is very valuable for the development of future interventions and for studies into the effectiveness of these interventions. Even though insight into the process is provided, some aspects of the paper, especially related to theoretical and empirical justification of the choices made, might require some attention or further elaboration. Please find below a list of specific points per step.

Thank you for your comments and suggestions. We have made a number of revisions based on the specific points you raise under each step below, which we feel provide additional clarity/elaborate on our choices made during the development process. The revisions we have made to address this point are detailed under each specific point you have raised below.

Step 1:

- The description of the planning group is clear and relevant stakeholders have been included. It might be good to reflect on the role and contributions of the stakeholders (What was there role in the planning process of the first four steps?).

Response:

We have provided additional detail of the expert panel, which was comprised of individuals who had prior experience of delivering air quality interventions. Their input in Stage 1 of the intervention mapping process informed the topic guides used for focus groups conducted in Stage 4. This is now clarified in the text.

- The literature reviews give insights into the different factors that play a role in this specific health problem. It might be good to create a Logic Model of the Problem which gives and add this as a file to the paper, as this provides a clear and logical ordering of all factors and determinants involved in the problem.

Response:

Thank you for your helpful suggestion. In response to Reviewer 1's comment (see page 1), we have now expanded our review of literature regarding interventions designed to reduce second-hand smoke exposure in the home in the introduction section of the paper, and we feel that this should assist the reader gain a clearer sense of the additional factors and determinants involved in creating and maintaining a smoke-free home. We have considered your suggestion to create a logic model of the problem, and we believe that for more complex needs assessments this would be a valuable means of adding clarity. However, we feel having expanded our review, that the factors and determinants involved in creating a smoke-free home should be clear to the reader.

Step 2:

- The matrices provide a clear overview of the performance (PO) and change objectives (CO) for this intervention. However, it might be advisable to start with stating the specific outcomes for the behavior of the target group. The main intervention aim is stressed in step 1, but it is not explained what the (SMART) outcomes for the two matrices were and, more importantly, how these were related to the main aim.

Response:

Thank you for your suggestion - we have now stated the SMART outcomes for both matrices, given detail as to how they were developed, and clarified how they relate to the main aim. 
- I was wondering whether the first matrix is focused on all parents, or on mothers only? In addition, the second matrix focusses on mothers only and is about making them responsible for the spread of the interventions to others in the household. It was not exactly clear to whether this should be an outcome of the intervention, or whether this is an implementation strategy for the intervention. In addition, from the text, it is not entirely clear why mothers are the best ones to take up this task?

Response:

These are very interesting points. The international literature on smoke-free homes has focused largely on behaviour change interventions for women and mothers as primary caregivers. There is a recognised gap in the literature with regard to father's experience related to creating/maintaining a smoke-free home. In our previous studies, we have mostly recruited mothers but a few fathers have taken part. Moreover, the literature also recognises that other household members should be involved in household attempts to create a smoke-free home to maximise the chances of success. On this basis, our first matrix is focused on parents (mothers, fathers, step-parents) rather than mothers only, and we have now clarified this in the text. The second matrix focuses on parents (mothers, fathers, step-parents), rather than mothers only being responsible for the spread of the intervention to other household members, and we have now amended this in the text. The spread of the intervention to others in the household is a desired outcome of the intervention.

- In line with this, here it might also be advisable to add a Logic Model of Change, as this provides a clear overview of all the outcomes involved.

Response:

Thanks for your suggestion, which we have considered carefully. However, we feel that a logic model would replicate the information provided already in the matrices. Perhaps if this were a more complicated set of issues then the addition of a logic model would be most useful. However, we feel that the matrices clearly set out the desired outcomes, and the steps required in order to achieve them.

- Even though the formulation of POs and COs is clear, it might be good to expand on the theoretical foundation. The Self-Determination Theory is used as the main theoretical framework for composing the POs. However, it is not directly clear how these specific objectives can be deduced from this theory. Moreover, the current formulation is more in line with regulation or planning models.

Response:

Thank you for questioning our theoretical foundation - we should have made it clearer that the determinants and change objectives in our matrices were developed on the basis of our literature review findings, the Self Determination Theory and Control Theory, We have now clarified this in the text, and included our rationale for using both theories as the framework for the study.

- The same holds for the specific determinants selected for the POs. It is highlighted that the most important and changeable determinants are selected, but it is advisable to reflect on the how this was done and which theoretical and/or empirical evidence was used here. By doing this, the reader knows that the most relevant determinants have indeed been selected in this specific example. 


\section{Response:}

This is a fair point. Given the number of POs, determinants and COs we have stopped short of setting out precisely which theoretical and/or empirical evidence was used to generate each one. However, we do take your point and we have therefore made it more explicit that all the POs, determinants and change objectives were derived either from existing literature, our review work, or based on Self Determination Theory and Control Theory as our main theories.

- Ideally, COs are stated as actions, which is not always the case in the current approach.

Response:

Thank you for pointing this out. We have reviewed the COs and ensured that they are now all stated as actions.

\section{Step 3}

- In step 3 the main methods are described. It is reflected that methods were selected based on theoretical considerations and empirical considerations (the review). It might be good to reflect on this. How is this linked to the results of the review?

\section{Response:}

We have revised this paragraph to include an example of one of our behaviour change intervention methods - planning coping responses - and how the decision to include this method is supported by recent literature outlining the importance of professionals being trained to develop skills on advising on smoke-free home related practical strategies, for example how to overcome barriers, to support households in creating a smoke-free home.

- For several COs within one determinant one specific method was selected. It might be good to reflect on why this was done. For instance, for self-efficacy, which is an important determinant, only the method 'planning coping responses' was selected. Does this adequately cover all action needed for self-efficacy part?

\section{Response:}

Table 3 shows examples of the methods selected for each determinant and its associated change objectives. We limited the number of examples given in this table so that the table did not become unwieldy. We have now added a sentence to clarify that the full list of behaviour change methods selected for each determinant and associated change objectives is presented in Table 4.

Step 4

The work for plan 4 has been well-described. In addition, several steps have been taken to develop the programme and several people have been involved. However, the methods listed in this table or not completely in line with the methods listed in the table for step 3 (e.g. personalized risk, goal setting).

Response: 
Thank you - we are pleased you feel that the work for Step 4 has been well described. The methods listed in Table 4 don't completely align with those outlined in Table 3 because Table 3 presents fewer, more detailed examples of the behaviour change methods selected. The full list is presented in Table 4.

Furthermore, some elements might benefit from elaboration:

- Can you elaborate on the procedures for the focus groups? Who was present, what did the interview guide look like, etc. This could be added

Response:

We have provided additional detail on the nature of the discussions that took place at the focus groups and during the interviews. We have also added text to clarify the procedures for the focus groups, and who was present. We have not added the interview/focus group topic guides to keep the length of the article more manageable, however we have included them as new supplementary materials.

- Who are the professionals that deliver the interventions to parents?

Response:

The abstract and introduction refer to health/care professionals, and we have provided a few examples of the types of roles this could include in the Introduction on page 4.

- How feasible are five hourly contact moments within a one-month period?

Response:

The health/care professionals that were involved in the development of this intervention generally see their clients on a weekly basis where support is required. However, the one-month period is an approximate time-frame and the intervention could be run across a slightly longer time span if required. We have now made this clear in the text on page 21.

- Did you also make use of other empirical studies to support the choices made? Most justification is based on the interviews, which is good, but the evidence from the literature is not clearly used here (while you did this beautiful review into interventions for this problem).

Response:

We did make use of the rapid review findings in Step 4, though most justification was based on interviews/focus groups as you say. We have however illustrated in the text where findings from the rapid reviews supported our choices made.

Discussion

In the discussion, you reflect very nicely on the effort done in step 3 and step 4. It might be good to also reflect on the first two steps (the Logic Models of the Problems and of Change), as this highlights the nature of the problem and the factors and determinants involved, as well as the proposed outcomes that will be targeted with your solution. In addition, although not part of this paper, it might be good to also reflect on the work that still will be done (e.g. step 5 and 6).

Response: 
Thank you for your comments. We have added a reflection on the first two steps of the intervention development programme as you suggest. We have refrained from reflecting on Steps 5 and 6 in the discussion, as earlier in the paper (p.4) we note that Steps 5 and 6 have been completed and published separately. 